\title{
Immunoglobulin G Measurement
}

National Cancer Institute

\section{Source}

National Cancer Institute. Immunoglobulin G Measurement. NCI Thesaurus. Code C81971.

The determination of the amount of immunoglobulin $\mathrm{G}$ present in a sample. 